\title{
First paediatric live donor liver transplant in Sri Lanka with 1 year outcome : challenges for the future
}

\author{
Rohan Siriwardana, Thilakarathne S, Fernando M, Gunetilleke M B, Weerasooriya A, Appuhamy C \\ Colombo North Centre for Liver Disease, University of Kelaniya, Sri Lanka
}

Keywords: Liver transplantation; child; live-donor; paediatric

\begin{abstract}
Introduction

Liver transplantation in the paediatric age group is demanding due to smaller body proportions and physiology. This paper describes the first successful paediatric liver transplant in Sri Lanka along with its one-year outcome. Describing the challenges faced during the process, we highlight the factors that need to be considered for a sustainable programme in the future.
\end{abstract}

\section{Methodology}

A 9-year-old girl who had progressive familial intra hepatic cholestasis type 3 was referred to us with features of end stage liver disease. She was identified as a suitable candidate for liver transplantation. Her 38-year-old mother was selected as the donor, who was evaluated for suitability of a left lateral segment donation.

\section{Results}

The first paediatric liver transplantation was performed in July 2020. The child's mother donated the left lateral segment weighing $325 \mathrm{~g}$. During the postoperative period the child developed outflow tract obstruction at the hepatic venous anastomosis. This was managed with a percutaneously placed stent. Six months after transplant, she developed an acute rejection that required steroids. Treatment of rejection was complicated with multiple liver abscesses caused by Aspergillus. The infection was treated with systemic antifungals and drainage. At one-year post transplant, the recipient had recovered from the trauma of surgery and had normal liver biochemistry, a patent hepatic venous stented anastomosis and complete resolution of the abscesses. We faced dual challenges in dealing with a live liver transplant donor and a young child who was the recipient. Our success, on this occasion, was underscored by the multidisciplinary contribution from specialists scattered across the island combined with state-public partnership.

Correspondence: Rohan Siriwardana

E-mail: rohansiriwardana@yahoo.com

(iDhttps://orcid.org/0000-0002-6477-2072

Received: 05-09-2021 Accepted: 20-11-2021

DOI: http://doi.org/10.4038/sljs.v39i3.8901

\section{Conclusion}

To offer a sustainable live donor liver transplant service for the future, many other aspects, beyond surgery itself, need to be addressed.

\section{Introduction}

Liver transplant [LT] is the ultimate cure for many paediatric liver diseases. Biliary atresia is the commonest primary aetiology requiring LT in children across the world. Among the rest, autoimmune liver disease, metabolic and genetic liver diseases such as Wilson disease, progressive familial intrahepatic cholestasis and liver malignancies predominate $[1,2]$. Paediatric liver transplant [PLT] is demanding due to the inherent nature of smaller body proportions. The history of paediatric liver transplant dates back many decades in affluent countries with over $85 \%$ five-year survival being reported worldwide $[3,4]$. This first successful paediatric liver transplant in Sri Lanka [SL] was performed in 2020, a decade after the first adult liver transplant was performed in SL [5]. We share our experience of the first PLT at the completion of the first post-transplant year while looking at how the future for a sustainable PLT programme could be achieved in the country.

\section{Presentation}

A 9-year-old girl from Jaffna, diagnosed with end-stage liver disease due to progressive familial intrahepatic cholestasis type 3 was referred for LT. She was diagnosed with chronic liver disease at two and a half years of age. Since the age of 8 , she had experienced several episodes of liver decompensation including hepatic encephalopathy, and was frequently hospitalised. Though she was identified as a candidate for LT, there was no paediatric liver transplant service existing in SL at that point of time. However at age 9 years, she was referred to the Colombo North Centre for Liver Diseases for consideration for liver transplant. On examination, she exhibited growth failure, was jaundiced and had early clubbing. Her liver function was impaired with INR, albumin and bilirubin being 2.35, $1.9 \mathrm{~g} / \mathrm{dL}$ and 169 micromol/L respectively. Following initial assessment and medical optimisation, she underwent comprehensive assessment for the suitability for LT. 
Following multidisciplinary discussion, she was listed for LT as she was identified as stable to withstand surgery in the absence of contraindications for LT.

\section{Assessment of the donor}

Following detailed evaluation, the child's 38 year old healthy mother with a BMI of $22 \mathrm{~kg} / \mathrm{m} 2$ was selected as the donor. Donor evaluation was carried out in two phases; initial assessment included the donor's vital organ assessment and psychological fitness for surgery. Next, liver anatomy and function were evaluated in detail. The liver attenuation index and fibro scan were used to exclude fatty liver. The donor's biliary anatomy showed favourable features for left lobe donation [6] - the segment IV biliary duct drained to the right side. The left duct was $2 \mathrm{~cm}$ in length. The left portal vein had a long extra hepatic path. The left hepatic artery was $3 \mathrm{~mm}$ in diameter and had a lower division. There were no sizable branches arising from left artery. The middle hepatic vein joined the left and the common trunk, which had an extrahepatic course. Segment II and III veins joined to give a 1.2 $\mathrm{cm}$ common opening. The total estimated left lateral section volume was $230 \mathrm{ml} \mathrm{[23 \%} \mathrm{of} \mathrm{the} \mathrm{total} \mathrm{donor} \mathrm{liver} \mathrm{volume].}$

\section{Surgery and follow-up}

Surgery was carried out in July 2020 at the Colombo North Teaching Hospital, Ragama. Specialists from the Lady Ridgway Hospital, National Hospital of Sri Lanka, Teaching Hospital Peradeniya and General Hospital Chilaw worked together as a team. The left lateral liver graft with part of segment IV weighing $325 \mathrm{~g}$ was harvested from the donor after 6-hours of surgery with a $250 \mathrm{ml}$ blood loss.

The native liver explantation took 4 hours with a blood loss of $175 \mathrm{ml}$. Total implantation took 40 minutes. Left hepatic veincaval anastomosis was performed with $6 / 0$ polypropylene without triangulation. Portal vein anastomosis was completed with $6 / 0$ polypropylene. The hepatic artery was anastomosed with $8 / 0$ polypropylene using visual magnification of 4.5 . Duct-to-duct biliary anastomosis was done with $6 / 0$ polydiaxone [figure 1]. Total surgery time was 8 hours. While the donor was managed at North Colombo following surgery, the recipient was extubated and transferred to a neighbouring private hospital for postoperative intensive care. The first 24 hours following operation was stormy and required high dose inotropic support, which was later tailed off.

The child continued to have high ascitic drainage in the postoperative period. Subsequently, doppler study and a hepatic venogram revealed hepatic venous outflow obstruction with a significant pressure gradient. Thus, on postoperative day 53, she underwent percutaneous stenting of the hepatic venous anastomosis by our interventional radiology team [figure 2].
Six months following surgery, the patient's liver function started deteriorating and a liver biopsy revealed moderate rejection. Rejection was managed successfully with methylprednisolone. With increased immunosuppression, the patient developed multifocal liver abscesses yielding Aspergillus species. The liver abscesses were treated successfully with antifungals and aspiration. At one year post transplant the child had good graft function and showed satisfactory catch-up growth.

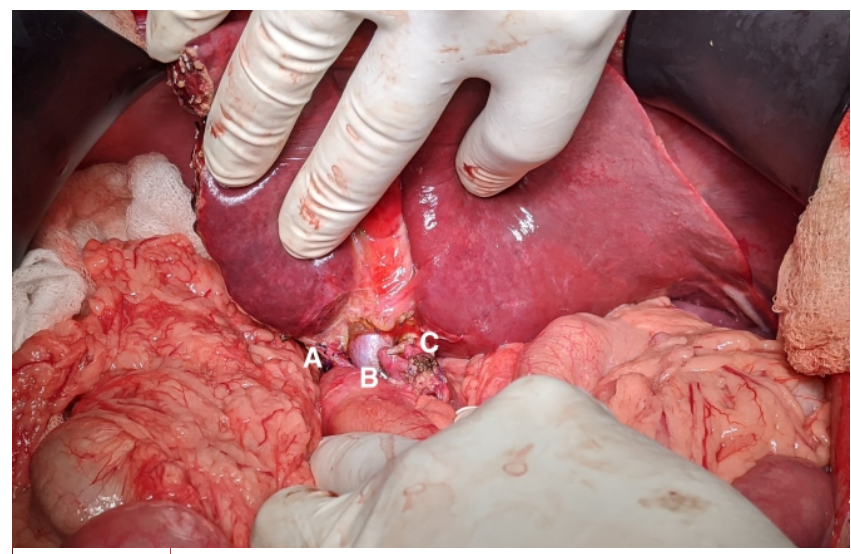

Figure 2. Photograph after the completion of implantation of left lateral section.

A - Bile duct B - Portal vein C- left hepatic artery

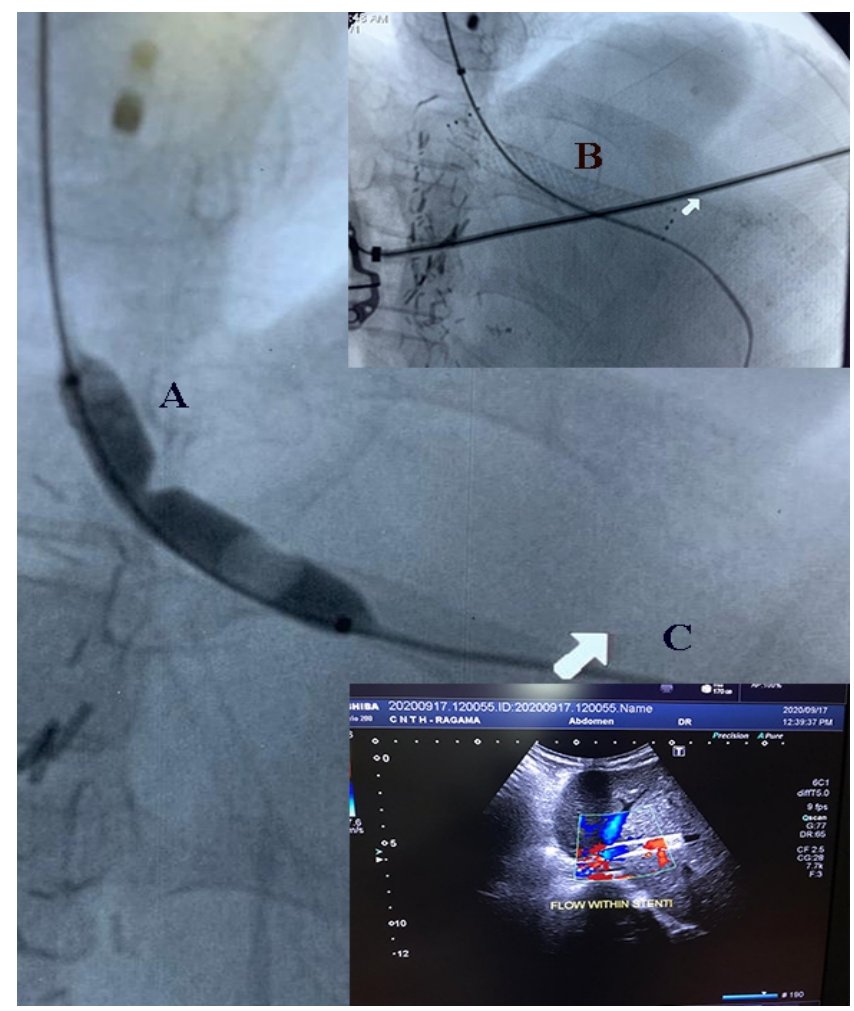

Figure 2.

A - fluoroscopy image showing the kink in the balloon placed across the left hepatic vein anastomosis,

B - stent placed across the anastomosis,

C - blood flow after placing the stent. 


\section{Discussion}

Liver transplantation is one of the most expensive surgical procedures, especially when recovery becomes protracted, like in our patient. The current estimated cost of liver transplant is between 10 and 12 million Sri Lankan rupees. Being a low middle-income country, a large majority of our patients cannot afford this financial burden. Transplant programs established in private institutions will not cater to the needs of the large majority of Sri Lankans. In India, alhough liver transplantation has flourished, all top-rated liver centres are run by private institutions, which cater to overseas patients and local patients with financial resources [7].

The child we report was from an extremely poor socioeconomic background, unable to afford private medical care. Though the surgery was performed in the non-fee levying state health sector, there were many other costs implications in the care of this patient; several trips from Jaffna to Colombo, lodging and treatment, especially during the time of protracted recovery. Fortunately, the junior members of our team who came to the fore, carried out an online patient support campaign to fund the child while the corporate hospital provided free postoperative intensive care. These multiple commercial support inputs eventually helped us overcome the challenges we faced after the transplant. A system that will utilize the goodwill of the general public and ensure continued support of the corporate sector will need to be fostered to fill the gap between demand and available free health care resources to make liver transplantation successful in selected public institutions for the benefit of the majority [8].

The first PLT was performed 10 years after the first successful adult liver transplant in SL [5] and reflects the challenges faced by a fledgling transplant program. The challenge was to bring together liver surgeons, adult and paediatric anaesthetists, paediatric hepatologists, interventional radiologists and liver-trained pathologists, who worked in different parts of the country, under one umbrella. At present, because there is no dedicated liver transplant institution, trained specialists are scattered in different parts of the country. Even though the first PLT was performed, the real challenge remains, which is to deliver a sustainable service. At present, for successful outcomes, it is essential for individuals of multiple disciplines to work in harmony and supporting each other. This report is a case in point. Eventually, with the increasing numbers of trained personnel and with greater experience, individual centres will become a flourishing reality.
Acknowledgements : The rest of the visiting members who contributed to the case were Dissanayake R, Kumarasinghe P, Dassanayake B, Pathmaperuma O, Liyanage C, Jayawickrema D, Ganewatte and postgraduate trainees in paediatrics, surgery and transplant anaesthesia.

All authors disclose no conflict of interest. The study was conducted in accordance with the ethical standards of the relevant institutional or national ethics committee and the Helsinki Declaration of 1975, as revised in 2000 .

\section{References}

1. Meng Q, Li N, Yuan L, Gao X. Analysis of common causes of liver damage among children 12 years and younger in Weifang. J Int Med Res. 2021 Apr;49[4] https://doi.org/10.1177/03000605211006661

2. Rawal N, Yazigi N.Pediatric Liver Transplantation. Pediatr Clin North Am. 2017 Jun;64[3]:677-684. https://doi.org/10.1016/j.pcl.2017.02.003

3. Kerkar N, Lakhole A. Pediatric liver transplantation: a North American perspective. Expert Rev Gastroenterol Hepatol. 2016 Aug; 10[8]:949-59 https://doi.org/10.1586/17474124.2016.1166951

4. Ng VL, Fecteau A, Shepherd R, Magee J, Bucuvalas J, Alonso E, et.al. Outcomes of 5-year survivors of pediatric liver transplantation: report on 461 children from a North American multicenter registry. Pediatrics. 2008 Dec;122[6]:e1128-35. https://doi.org/10.1542/peds.2008-1363

5. Wijeyaratne SM, Gunawansa N, Cassim MRN, Abayadeera A. The first successful liver transplants in Sri Lanka. The CMJ.2011; $56[2], 81-82$ https://doi.org/10.4038/cmj.v56i2.2381

6. Soejima Y, Shimada M, Suehiro T, Hiroshige S, Ninomiya M, Shiotani S, Harada N, Hideki I, Yonemura Y, Maehara Y. Outcome analysis in adult-to-adult living donor liver transplantation using the left lobe. Liver Transpl. 2003;9:581-58 https://doi.org/10.1053/jlts.2003.50114

7. Nagral S, Nanavathi A, Nagral A, Liver Transplantation in India: At the Crossroads. J Clin Exp Hepatol. 2015 Dec; 5[4]: 329-40. https://doi.org/10.1016/j.jceh.2015.11.001

8. Saigal S., Shah S.R. Liver transplantation-Economics in the less developed world. Indian J Gastroenterol, 31, 13-14 [2012]. https://doi.org/10.1007/s12664-011-0159-8 